\title{
EXTENDED TORELLI MAP TO THE IGUSA BLOWUP IN GENUS 6, 7, AND 8
}

\author{
VALERY ALEXEEV, RYAN LIVINGSTON, JOSEPH TENINI, MAXIM ARAP, XIAOYAN \\ HU, LAUREN HUCKABA, PATRICK MCFADDIN, STACY MUSGRAVE, JAEHO SHIN, \\ AND CATHERINE ULRICH
}

\begin{abstract}
It was conjectured in [Nam73] that the Torelli map $\mathrm{M}_{g} \rightarrow \mathrm{A}_{g}$ associating to a curve its jacobian extends to a regular map from the DeligneMumford moduli space of stable curves $\overline{\mathrm{M}}_{g}$ to the (normalization of the) Igusa blowup $\overline{\mathrm{A}}_{g}^{\text {cent }}$. A counterexample in genus $g=9$ was found in [AB11]. Here, we prove that the extended map is regular for all $g \leq 8$, thus completely solving the problem in every genus.
\end{abstract}

\section{Introduction}

The Torelli map $\mathrm{M}_{g} \rightarrow \mathrm{A}_{g}$ associates to a smooth curve $C$ its jacobian $J C$, a principally polarized abelian variety. Does it extend to a regular map $\overline{\mathrm{M}}_{g} \rightarrow \overline{\mathrm{A}}_{g}$, where $\overline{\mathrm{M}}_{g}$ is Deligne-Mumford's moduli space of stable curves, and $\overline{\mathrm{A}}_{g}$ is a toroidal compactification of $\mathrm{A}_{g}$ ?

This question was first asked in a pioneering paper of Namikawa [Nam73] in the case when $\overline{\mathrm{A}}_{g}=\overline{\mathrm{A}}_{g}^{\text {cent }}$ is the normalization of the Igusa blowup $\mathrm{Bl}_{\partial A_{g}^{*}} A_{g}^{*}$ of the Satake compactification along the boundary. This compactification was introduced by Igusa in [Igu67], and is possibly the first toroidal compactification ever constructed. It corresponds to the central cone decomposition.

Namikawa conjectured that the extended map is always regular. He was able to prove it for the stable curves with a planar dual graph, and for curves of low genus; the case $g \leq 6$ was stated without proof. (Note: the graphs in this paper may have multiple edges and loops).

The question was recently revisited in [AB11], who showed the following:

(1) Let $C$ be a stable curve of genus $g$, and let $\Gamma$ be its dual graph. Then the rational map $\overline{\mathrm{M}}_{g} \rightarrow \overline{\mathrm{A}}_{g}^{\mathrm{cent}}$ is regular in a neighborhood of the point $[C] \in \overline{\mathrm{M}}_{g} \Longleftrightarrow$ there exists a positive definite integral-valued quadratic form $q$ on the first cohomology $H^{1}(\Gamma, \mathbb{Z})$ such that $q\left(e_{i}^{*}\right)=1$ for every non-bridge edge $e_{i}$ of $\Gamma$. Such quadratic forms $q$ are called integral edge-minimizing metrics or $\mathbb{Z}$-emms for short.

Recall that for a graph, $H^{1}(\Gamma)=C^{1}(\Gamma) / d C^{0}(\Gamma)$, where $C^{1}(\Gamma, \mathbb{Z})=\oplus_{\text {edges }} e_{i} \mathbb{Z} e_{i}^{*}$, $C^{0}(\Gamma, \mathbb{Z})=\oplus_{\text {vertices } v_{j}} \mathbb{Z} v_{j}^{*}$, and $d v_{j}^{*}=\sum_{e_{i} \text { begins with } v_{j}} e_{i}^{*}-\sum_{e_{i} \text { ends with } v_{j}} e_{i}^{*}$. We denote the image of $e_{i}^{*}$ in $H^{1}(\Gamma, \mathbb{Z})$ by the same letter $e_{i}^{*}$ and call it a coedge.

(2) Call a graph cohomology-irreducible if there does not exist a partition of its edges into two groups $I_{1} \sqcup I_{2}$ such that $H^{1}(\Gamma, \mathbb{Z})=\left\langle e_{i}^{*}, i \in I_{1}\right\rangle \oplus\left\langle e_{i}^{*}, i \in I_{2}\right\rangle$. Then

Date: May 14, 2011. 
$\Gamma$ is either a simple loop (a graph with one vertex and one edge), or $\Gamma$ is loopless and 2-connected.

For every graph $\Gamma$, one has $H^{1}(\Gamma, \mathbb{Z})=\oplus H^{1}\left(\Gamma_{k}, \mathbb{Z}\right)$ for some cohomologyirreducible graphs $\Gamma_{k}$ and all coedges $e_{i}^{*}$ lie in the direct summands. We call $G_{k}$ cohomology-irreducible components of $\Gamma$. Then there exists a $\mathbb{Z}$-emm for $\Gamma \Longleftrightarrow$ there exist $\mathbb{Z}$-emms for all $\Gamma_{k}$.

(3) If a graph $\Gamma$ is cohomology-irreducible and $q$ is a $\mathbb{Z}$-emm for $\Gamma$ then the lattice $\left(H^{1}(\Gamma, \mathbb{Z}), 2 q\right)$ is a root lattice of type $A_{g}, D_{g}(g \geq 4)$, or $E_{g}(g=6,7,8)$. Further, there exists a $\mathbb{Z}$-emm of type $A_{g} \Longleftrightarrow \Gamma$ is planar, and for $g \geq 4$ there exists a $\mathbb{Z}$-emm of type $D_{g} \Longleftrightarrow \Gamma$ is projective planar, i.e. can be embedded into the projective plane $P=\mathbb{R P}^{2}$.

The famous theorem of Kuratowski says that a graph is non-planar iff it contains a subgraph homeomorphic either to $K_{5}$ or to $K_{3,3}$. A Kuratowski-type theorem for the projective plane $P$ was proved by Archdeacon [Arc81, Arc80] who showed that the list of 103 minimal non-projective planar graphs produced earlier by GloverHuneke-Wang [GHW79] is complete; any other non-projective planar graph contains a subgraph homeomorphic to one of them. The smallest graph on their list has genus 6 .

This implies that every graph of genus $\leq 5$ has a $\mathbb{Z}$-emm, and consequently the extended Torelli map $\overline{\mathrm{M}}_{g} \rightarrow \overline{\mathrm{A}}_{g}^{\mathrm{cent}}$ is regular for $g \leq 5$. On the other hand, as [AB11] notes, there exist cohomology-irreducible non-projective planar graphs of genus 9 , so the extended Torelli map is not regular for every $g \geq 9$.

Here are the main results of this paper:

Theorem 1.1. Let $\Gamma$ be a cohomology-irreducible non-projectively planar graph of genus $g=6,7$, or 8 . Then $\Gamma$ admits a $\mathbb{Z}$-emm of type $E_{g}$.

Corollary 1.2. The extended Torelli map $\overline{\mathrm{M}}_{g} \rightarrow \overline{\mathrm{A}}_{g}^{\mathrm{cent}}$ is regular for $g \leq 8$.

Corollary 1.3. Let $C$ be a stable curve of genus $g$, and $\Gamma$ be its dual graph. Then the extended Torelli map $\overline{\mathrm{M}}_{g} \rightarrow \overline{\mathrm{A}}_{g}^{\mathrm{cent}}$ is regular in a neighborhood of the point $[C] \in \overline{\mathrm{M}}_{g} \Longleftrightarrow$ every cohomology-irreducible component $\Gamma_{k}$ has genus $\leq 8$ or is a projectively planar graph of genus $\geq 9$.

The plan of the paper is as follows. In Section 2, we reduce the proof of Theorem 1.1 to checking finitely many graphs: one graph for $g=6,14$ graphs for $g=7$, and 2394 graphs for $g=8$. In Section 3, we give a finite algorithm for an arbitrary graph, and then run it for the only graph needed in genus 6 . In Section 4 , we give the 14 graphs in genus 7 that have to be checked, and explicitly list a $\mathbb{Z}$-emm for each of them. In Section 5, we state our computer-aided findings for genus 8 .

Acknowledgments. The subject of the paper was one of the topics of a VIGRE research group at the University of Georgia in the Fall of 2010, led by the first author. We would like to acknowledge NSF's VIGRE support under DMS-0738586. We thank Boris Alexeev for writing an alternative code for finding $\mathbb{Z}$-emms for a graph. 


\section{Reduction to finitely many graphs}

As noted in [AB11, Sec.2], for the proof of Theorem 1.1 we may reduce to graphs which are trivalent. So let $H$ be a cohomology-irreducible non-projectively planar trivalent graph of genus $g=6,7$ or 8 . One says that $H$ is irreducible with respect to $P$ if $H$ does not embed into $P$, but for any edge $e$ in $H, H-e$ does embed into $P$. We now describe a process which will reduce $H$ to a trivalent graph irreducible w.r.t. $P$. The operations (3a), (3b), (3c) are illustrated in Figures 2, 3, 4.

(1) If the graph is irreducible w.r.t. $P$, stop and call this graph $H^{\prime}$.

(2) If not, choose an edge $e$ so that $H-e$ does not embed into $P$ and delete $e$ from the graph.

(3a) If $e$ was not a loop and did not have a parallel edge, then, denoting by $v_{1}$ and $v_{2}$ the distinct vertices to which $e$ is incident, contract an edge incident to $v_{1}$ and an edge incident to $v_{2}$.

(3b) If $e$ was not a loop but had a parallel edge $f$, then, denoting by $v_{1}$ and $v_{2}$ the distinct vertices to which $e$ and $f$ are incident, contract the edge incident to $v_{1}$ and different from $f$ and the edge incident to $v_{2}$ and different from $f$.

(3c) If $e$ was a loop incident to $v$, then delete the remaining edge $f$ incident to $v$ and, denoting by $w$ the other vertex to which $f$ is incident, contract one of the other two edges incident to $w$ and different from $f$.

Notice that the above operations (3a)-(3b)-(3c) drop the genus of the graph by 1 except for operation (3a) when e is a bridge. Repeating this process we get a graph $H^{\prime}$ irreducible w.r.t. $P$ which is of the form $H^{\prime}=\widetilde{H} \cup\left\{u_{1}, \ldots, u_{k}\right\}$ where the $u_{i}$ are isolated vertices and $\widetilde{H}$ is a trivalent graph irreducible w.r.t. $P$. By [GH75, Mil73] (see also [Arc81, Arc80]), $\widetilde{H}$ is isomorphic to one of the following:

(i) The connected graph $G$ of genus 6 shown in Figure 5.

(ii) The connected graphs $F_{11}, F_{12}, F_{13}, F_{14}$ of genus 7 shown in Figures 6-9.

(iii) The graph $E_{42}$ shown in Figure 1.
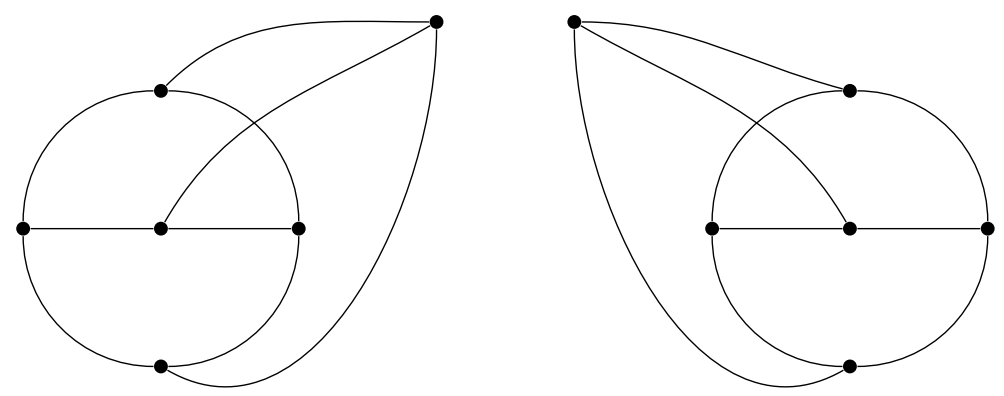

Figure 1. The Graph $E_{42}$.

Thus, we may construct $H$ from $\widetilde{H}$ by reversing the algorithm above. We make this explicit for the relevant genera 6,7 and 8 . 
2.1. $H$ has genus 6. Since $H$ is cohomology-irreducible, it has no bridges and so operations (3a), (3b) and (3c) would all drop the genus. Thus $H$ is already irredicible with respect to $P$ and so $H=\widetilde{H}=G$. Thus, to show the existence of $\mathbb{Z}$-emms for graphs of genus 6 , it suffices to produce one for $G$.

2.2. $H$ has genus 7. Either $\widetilde{H}$ equals one of $F_{11}, F_{12}, F_{13}, F_{14}$ or $\widetilde{H}=G$. In the first case we have that $H$ is equal to one of $F_{11}, F_{12}, F_{13}, F_{14}$ (again since $H$ was cohomology-irreducible, thus bridgeless). The second case is slightly more complicated. First notice that $H^{\prime}$ has at most one isolated vertex $v$, because in the case of applying (3c), the genus drops by 1 . Then $H$ may be obtained from $\widetilde{H}$ by doing one of the following three operations. Notice that (a), (b) and (c) are the inverse operations of (3a), (3b) and (3c) (defined above) respectively.

(a) Choose two distinct edges $e_{1}$ and $e_{2}$ and add an edge from the midpoint of $e_{1}$ to the midpoint of $e_{2}$.

(b) Choose an edge and add a handle to it.

(c) Choose an edge $e^{\prime}$ and add an edge $f$ from the midpoint of $e^{\prime}$ to the isolated vertex $v$. Then add a loop $e$ to $v$.

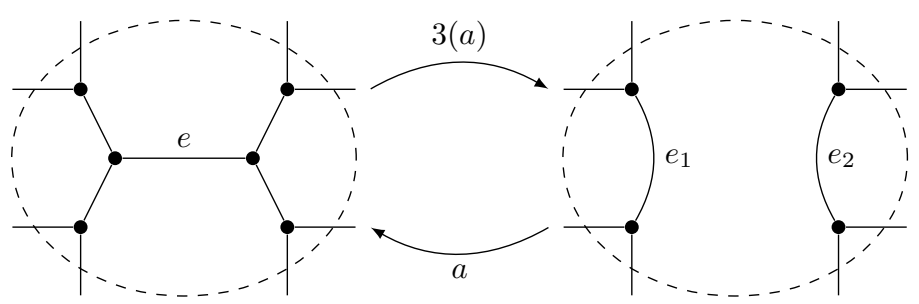

Figure 2. The procedures (3a) and (a).

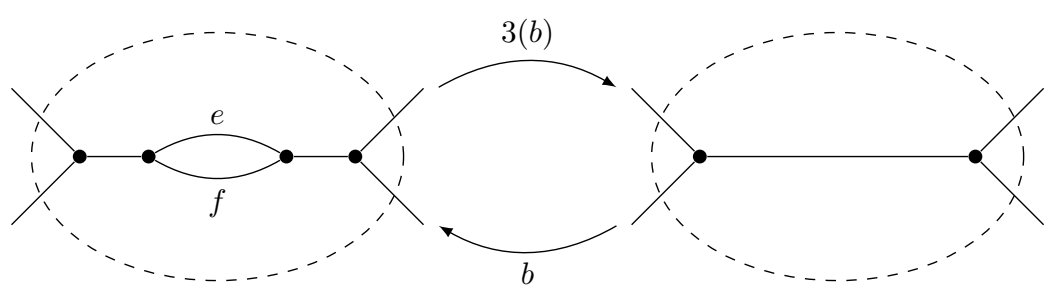

Figure 3 . The procedures (3b) and (b).

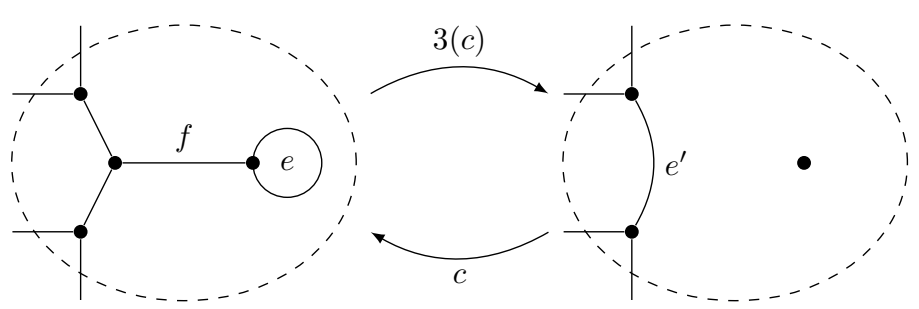

Figure 4. The procedures (3c) and (c). 
In the case (c), $f$ is a bridge and so we do not need to consider graphs acquired from $\widetilde{H}$ from operation (c).

A careful but elementary analysis shows that the cases (a) and (b), up to symmetries, produce ten possible graphs for $H$. We denote these graphs $G_{1}, \ldots G_{10}$, they appear in figures 10-19 below. Thus, to show the existence of $\mathbb{Z}$-emms for graphs of genus 7 it suffices to produce one for $F_{11}, F_{12}, F_{13}, F_{14}$ and $G_{i}$ for $i \in\{1, \ldots, 10\}$.

2.3. $H$ has genus 8. Since $H$ is cohomology-irreducible, the graphs $H$ and $\widetilde{H}$ can not be isomorphic to $E_{42}$ : otherwise, $H$ would have genus $\geq 10$.

We may choose an edge $e$ so that $H-e$ does not embed into $P$. Since $e$ is not a bridge, we may construct a trivalent graph $\operatorname{Simp}(H-e)$ from $H-e$ by contracting edges which were incident to $e$, as in $(3 \mathrm{a})$ or $(3 \mathrm{~b})$. So $\operatorname{Simp}(H-e)$ is a trivalent graph of genus 7 which does not embed into $P$. Hence by our above argument, $\operatorname{Simp}(H-e)$ is isomorphic to one of $F_{11}, F_{12}, F_{13}, F_{14}, G_{i}$ for $i \in\{1, \ldots, 10\}$, or a graph $G^{\prime}$ obtained from $G$ by choosing an edge $e^{\prime}$, adding an edge $f$ from the midpoint of $e^{\prime}$ to an isolated vertex $v$ and then adding a loop $e$ to $v$, as in (c).

In the latter case, $H$ is obtained from the graph $G$ by performing operation (c) and then (a). But, equivalently, this can be accomplished by the operations (a) and then (b). Thus, to prove Theorem 1.1 for $g=8$, it is sufficient to find $\mathbb{Z}$-emms for the finitely many graphs obtained from one of the graphs $F_{11}-F_{14}, G_{1}-G_{10}$ by performing one operation of type (a) or (b).

\section{Genus 6}

In this section, we explain the general method for finding a $\mathbb{Z}$-emm for any graph, and illustrate it in the case of the trivalent genus 6 graph $G$.

3.1. Procedure for a general graph. Let $\Gamma$ be a directed graph of genus $g$ with edge set $E=\left\{e_{1}, \ldots, e_{n}\right\}$. After renaming the edges, we may insist that the edges $\left\{e_{g+1}, \ldots, e_{n}\right\}$ induce a spanning tree $T$ of $\Gamma$. Then for each $e_{i}$ with $i \in\{1, \ldots, g\}$, we have a corresponding basis element $f_{i}$ of the homology group $H_{1}(\Gamma, \mathbb{Z})$, given by:

$$
f_{i}=e_{i}+\sum_{e_{s} \in T} b_{i, s} e_{s}, \quad b_{i, s}=0, \pm 1, i \in\{1, \ldots, g\},
$$

and the coedges $e_{1}^{*}, \ldots, e_{g}^{*}$ form a basis of the cohomology group $H^{1}(G, \mathbb{Z}$ ) (cf. [AB11, Lemma 2.3]).

Specifically, $f_{i}$ is given by the unique simple cycle in $\Gamma$ which uses only the edge $e_{i}$ and edges of $T$. If we write the vectors $f_{i}$ as the rows of a $g \times n$ matrix then the columns of this matrix are the coedges $e_{i}^{*} \in H^{1}(G, \mathbb{Z})$ written in the basis $\left\{e_{1}^{*}, \ldots, e_{g}^{*}\right\}$. In particular, the first $g$ columns form an identity matrix.

Let $q$ be a $\mathbb{Z}$-emm for $\Gamma$. Since $q$ is a $\mathbb{Z}$-valued quadratic form, we may associate to $q$ an even integral matrix $M_{q}=\left(a_{i, j}\right)$ such that

$$
q\left(x_{1}, \ldots, x_{g}\right)=\left(x_{1}, \ldots, x_{g}\right) \frac{1}{2} M_{q}\left(x_{1}, \ldots, x_{g}\right)^{T} .
$$

Note here that $a_{i, j}=a_{j, i}$ is just the coefficient of the term $x_{i} x_{j}$ in $q\left(x_{1}, \ldots, x_{g}\right)$ if $i \neq j$ and $a_{i, i}$ is just twice the coefficient of the term $x_{i}^{2}$ in $q\left(x_{1}, \ldots, x_{g}\right)$. 
We need to enforce the condition that $q\left(e_{i}^{*}\right)=1$ for $i=1, \ldots, n$. To ensure that $q\left(e_{i}^{*}\right)=1$ for $i=1, \ldots, g$ we must have $a_{i, i}=2$. Now we must ensure that $q\left(e_{i}^{*}\right)=1$ for $i=g+1, \ldots, n$. This is equivalent to $n-g$ linear equations on $a_{i, j}$ :

$$
1=\sum_{i=1}^{g} c_{i}^{2}+\sum_{1 \leq i<j \leq g} c_{i} c_{j} a_{i, j} \quad \text { for each column }\left(c_{i}\right) .
$$

Further, the condition that $q$ is positive definite implies that each $a_{i, j} \in\{0, \pm 1\}$. Thus, for any given graph, we reduced the problem to a finite computation.

3.2. Computation for graph $G$. We now specialize to graph $G$. In Figure 5 it is shown as a labeled directed graph with a spanning tree denoted by bold edges.

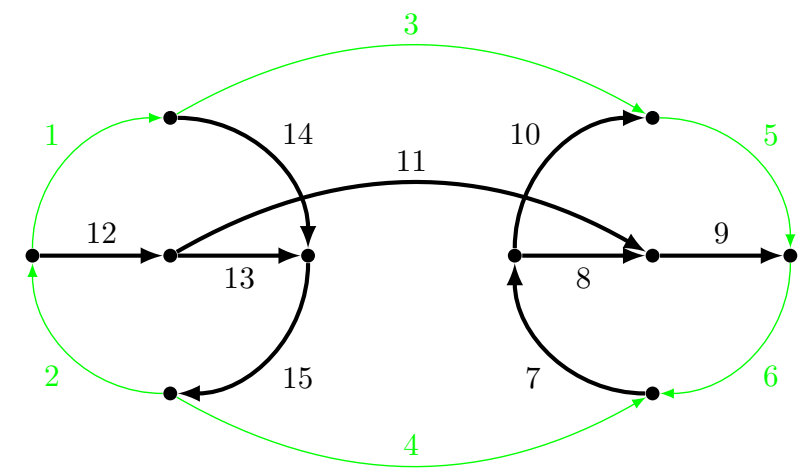

Figure 5. The Graph $G$.

Using the spanning tree drawn and the process described above we get a basis for $H_{1}(G, \mathbb{Z})$, written as the rows of the following matrix:

\begin{tabular}{c|c|c|c|c|c|c|c|c|c|c|c|c|c|c|c} 
& $e_{1}$ & $e_{2}$ & $e_{3}$ & $e_{4}$ & $e_{5}$ & $e_{6}$ & $e_{7}$ & $e_{8}$ & $e_{9}$ & $e_{10}$ & $e_{11}$ & $e_{12}$ & $e_{13}$ & $e_{14}$ & $e_{15}$ \\
\hline$f_{1}$ & 1 & 0 & 0 & 0 & 0 & 0 & 0 & 0 & 0 & 0 & 0 & -1 & -1 & 1 & 0 \\
$f_{2}$ & 0 & 1 & 0 & 0 & 0 & 0 & 0 & 0 & 0 & 0 & 0 & 1 & 1 & 0 & 1 \\
$f_{3}$ & 0 & 0 & 1 & 0 & 0 & 0 & 0 & 1 & 0 & -1 & -1 & 0 & 1 & -1 & 0 \\
$f_{4}$ & 0 & 0 & 0 & 1 & 0 & 0 & 1 & 1 & 0 & 0 & -1 & 0 & 1 & 0 & 1 \\
$f_{5}$ & 0 & 0 & 0 & 0 & 1 & 0 & 0 & -1 & -1 & 1 & 0 & 0 & 0 & 0 & 0 \\
$f_{6}$ & 0 & 0 & 0 & 0 & 0 & 1 & 1 & 1 & 1 & 0 & 0 & 0 & 0 & 0 & 0
\end{tabular}

The linear equations become:

(1) $1=2+a_{4,6}$

(2) $1=4+a_{3,4}-a_{3,5}+a_{3,6}-a_{4,5}+a_{4,6}-a_{5,6}$

(3) $1=2-a_{5,6}$

(4) $1=2-a_{3,5}$

(5) $1=2+a_{3,4}$

(6) $1=2-a_{1,2}$

(7) $1=4-a_{1,2}-a_{1,3}-a_{1,4}+a_{2,3}+a_{2,4}+a_{3,4}$

(8) $1=2-a_{1,3}$

(9) $1=2+a_{2,4}$ 
So, equations (1),(3),(4),(5),(6),(8),(9) immediately imply that $1=a_{5,6}=a_{3,5}=$ $a_{1,2}=a_{1,3}$ and $-1=a_{4,6}=a_{3,4}=a_{2,4}$. Applying this information to (2) and (7) we get $1=a_{3,6}-a_{4,5}$ and $1=a_{2,3}-a_{1,4}$ respectively. Let us arbitrarily choose $a_{3,6}=a_{2,3}=1$ and $a_{4,5}=a_{1,4}=0$. Hence, we will get a $\mathbb{Z}$-emm if we can choose the remaining terms of the below matrix in such a way that it is positive definite.

$$
\left(\begin{array}{cccccc}
2 & 1 & 1 & 0 & a_{1,5} & a_{1,6} \\
1 & 2 & 1 & -1 & a_{2,5} & a_{2,6} \\
1 & 1 & 2 & -1 & 1 & 1 \\
0 & -1 & -1 & 2 & 0 & -1 \\
a_{5,1} & a_{5,2} & 1 & 0 & 2 & 1 \\
a_{6,1} & a_{6,2} & 1 & -1 & 1 & 2
\end{array}\right)
$$

One such choice is to set all the unknowns to 0 . Then the quadratic form corresponding to this matrix is:

$$
\begin{aligned}
q\left(x_{1}, x_{2}, x_{3}, x_{4}, x_{5}, x_{6}\right)= & x_{1}^{2}+x_{1} x_{2}+x_{1} x_{3}+x_{2}^{2}+x_{2} x_{3}- \\
& x_{2} x_{4}+x_{3}^{2}-x_{3} x_{4}+x_{3} x_{5}+x_{3} x_{6}+ \\
& x_{4}^{2}-x_{4} x_{6}+x_{5}^{2}+x_{5} x_{6}+x_{6}^{2}
\end{aligned}
$$

One can easily check by diagonalizing that this quadratic form is indeed positive definite. Moreover, in an appropriately chosen basis, it is isomoprhic to the standard quadratic form $E_{6}$.

\section{Genus 7}

We repeat the general procedure of the previous section for the graphs $F_{11}-F_{14}$ and $G_{1}-G_{10}$. Below, we list one explicit $\mathbb{Z}$-emm for each of these graphs. The detailed computations are available in the long version of this paper.

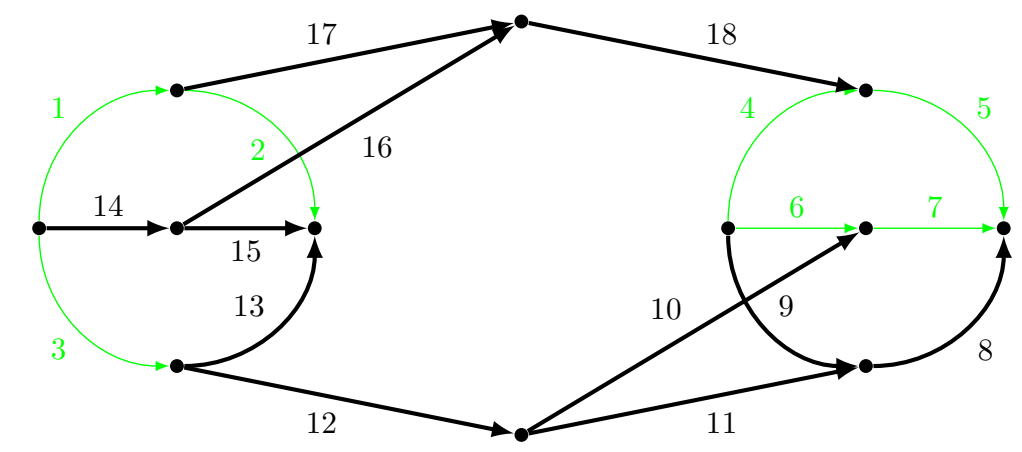

Figure 6 . The Graph $F_{11}$.

$$
\begin{aligned}
q_{F_{11}}\left(x_{1}, x_{2}, x_{3}, x_{4}, x_{5}, x_{6}, x_{7}\right)= & x_{1}^{2}+x_{1} x_{2}-x_{1} x_{3}-x_{1} x_{6}-x_{1} x_{7}+x_{2}^{2}+ \\
& x_{2} x_{4}-x_{2} x_{6}+x_{3}^{2}+x_{3} x_{4}+x_{3} x_{7}+x_{4}^{2}+ \\
& x_{4} x_{5}-x_{4} x_{6}+x_{5}^{2}-x_{5} x_{6}-x_{5} x_{7}+ \\
& x_{6}^{2}+x_{6} x_{7}+x_{7}^{2}
\end{aligned}
$$




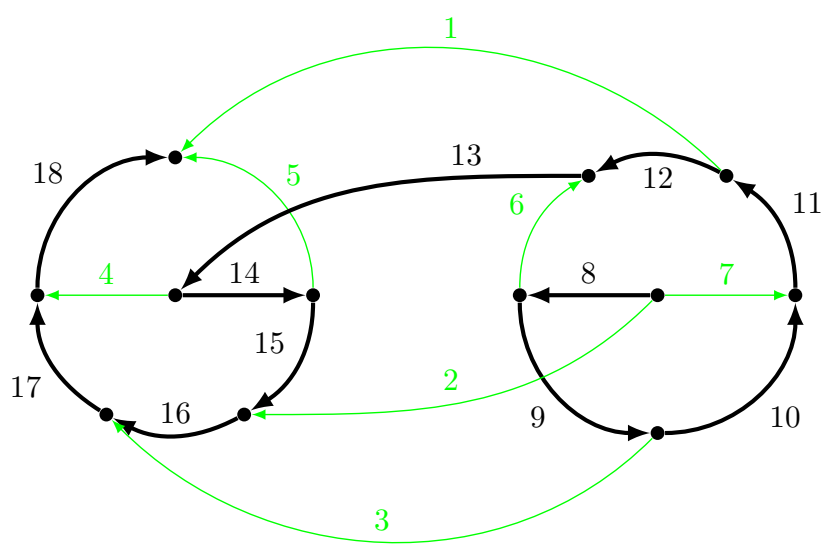

Figure 7 . The graph $F_{12}$.

$$
\begin{aligned}
q_{F_{12}}\left(x_{1}, x_{2}, x_{3}, x_{4}, x_{5}, x_{6}, x_{7}\right)= & x_{1}^{2}-x_{1} x_{3}-x_{1} x_{5}+x_{2}^{2}-x_{2} x_{3} \\
& -x_{2} x_{4}+x_{2} x_{5}-x_{2} x_{7}+x_{3}^{2}-x_{3} x_{6}+x_{3} x_{7}+ \\
& x_{4}^{2}-x_{4} x_{5}+x_{5}^{2}+x_{6}^{2}-x_{6} x_{7}+x_{7}^{2}
\end{aligned}
$$

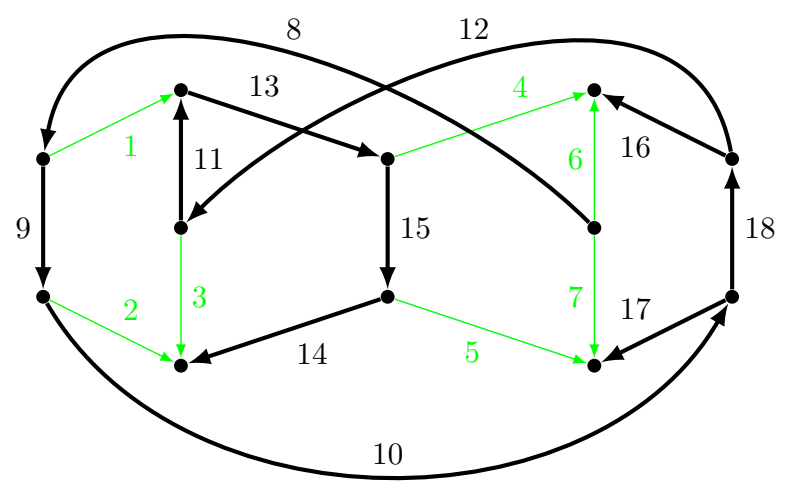

Figure 8 . The Graph $F_{13}$

$$
\begin{aligned}
q_{F_{13}}\left(x_{1}, x_{2}, x_{3}, x_{4}, x_{5}, x_{6}, x_{7}\right)= & x_{1}^{2}+x_{1} x_{5}-x_{1} x_{7}+x_{2}^{2}-x_{2} x_{3}+ \\
& x_{2} x_{5}-x_{2} x_{6}+x_{3}^{2}+x_{4}^{2}-x_{4} x_{5}- \\
& x_{4} x_{6}+x_{4} x_{7}+x_{5}^{2}-x_{5} x_{7}+x_{6}^{2}- \\
& x_{6} x_{7}+x_{7}^{2}
\end{aligned}
$$




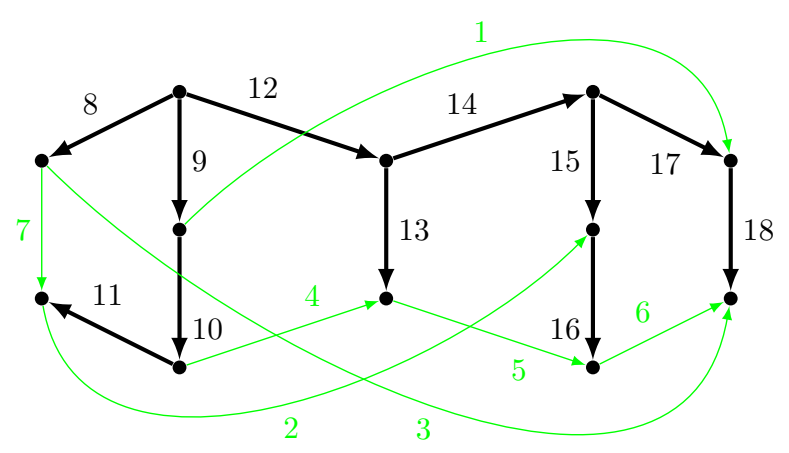

Figure 9 . The Graph $F_{14}$
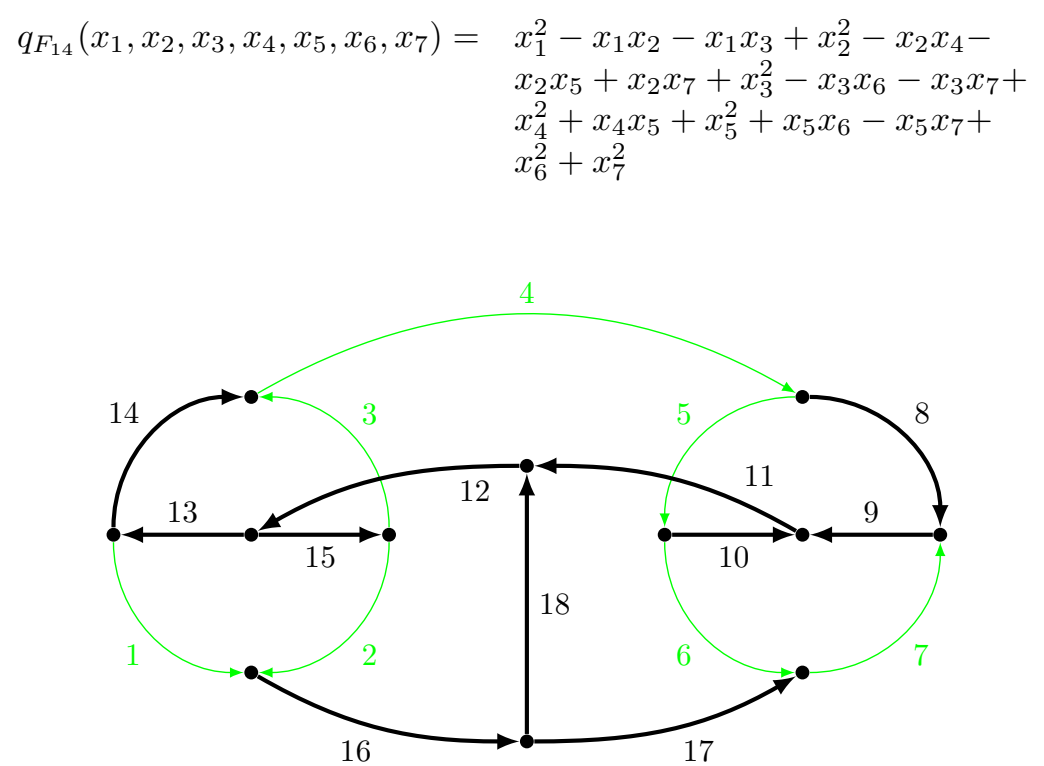

Figure 10. The graph $G_{1}$.

$$
\begin{aligned}
q_{G_{1}}\left(x_{1}, x_{2}, x_{3}, x_{4}, x_{5}, x_{6}, x_{7}\right)= & x_{1}^{2}-x_{1} x_{2}+x_{1} x_{3}+x_{1} x_{5}+x_{1} x_{6}+ \\
& x_{1} x_{7}+x_{2}^{2}-x_{2} x_{3}-x_{2} x_{4}-x_{2} x_{5}- \\
& x_{2} x_{6}+x_{3}^{2}+x_{3} x_{4}+x_{3} x_{5}+x_{3} x_{6}+ \\
& x_{4}^{2}+x_{4} x_{5}-x_{4} x_{7}+x_{5}^{2}+x_{5} x_{6}+ \\
& x_{6}^{2}+x_{6} x_{7}+x_{7}^{2}
\end{aligned}
$$




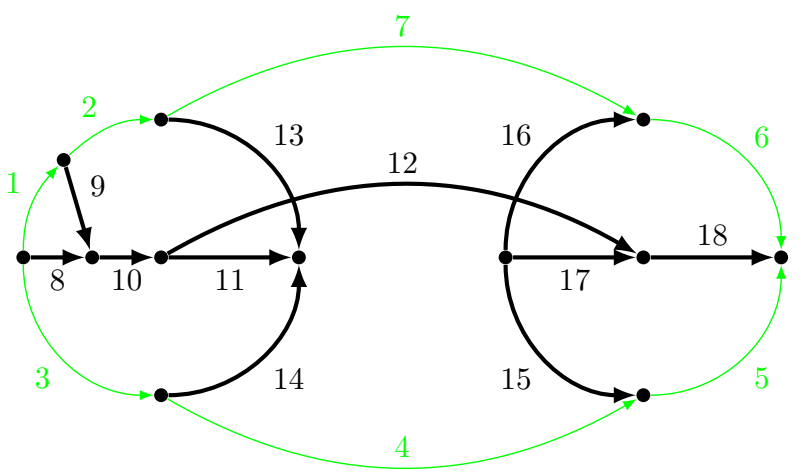

Figure 11 . The graph $G_{2}$.

$$
\begin{aligned}
q_{G_{2}}\left(x_{1}, x_{2}, x_{3}, x_{4}, x_{5}, x_{6}, x_{7}\right)= & x_{1}^{2}+x_{1} x_{2}-x_{1} x_{3}+x_{2}^{2}-x_{2} x_{3}- \\
& x_{2} x_{4}+x_{2} x_{7}+x_{3}^{2}+x_{3} x_{4}+x_{4}^{2}+ \\
& x_{4} x_{5}-x_{4} x_{6}-x_{4} x_{7}+x_{5}^{2}-x_{5} x_{6}+ \\
& x_{6}^{2}+x_{6} x_{7}+x_{7}^{2}
\end{aligned}
$$

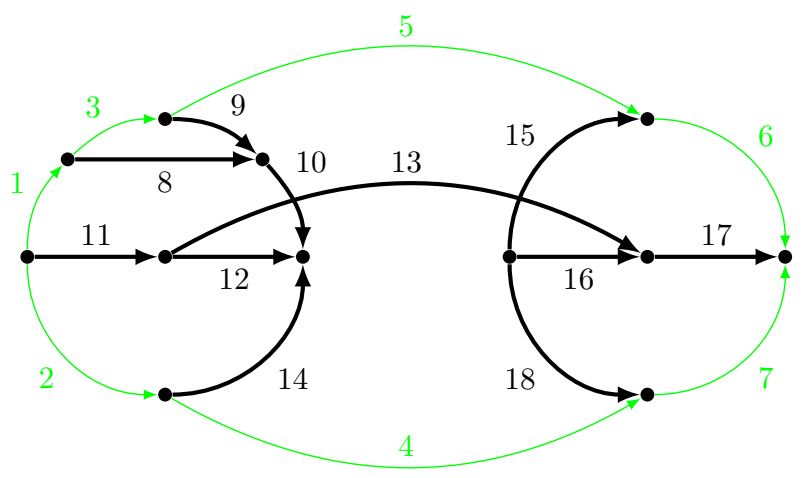

Figure 12. The Graph $G_{3}$

$$
\begin{aligned}
q_{G_{3}}\left(x_{1}, x_{2}, x_{3}, x_{4}, x_{5}, x_{6}, x_{7}\right)= & x_{1}^{2}-x_{1} x_{2}+x_{1} x_{3}-x_{1} x_{4}+x_{1} x_{5}+ \\
& x_{2}^{2}+x_{2} x_{4}+x_{3}^{2}+x_{3} x_{5}+x_{4}^{2}- \\
& x_{4} x_{5}-x_{4} x_{6}+x_{4} x_{7}+x_{5}^{2}+x_{5} x_{6}+ \\
& x_{6}^{2}-x_{6} x_{7}+x_{7}^{2}
\end{aligned}
$$




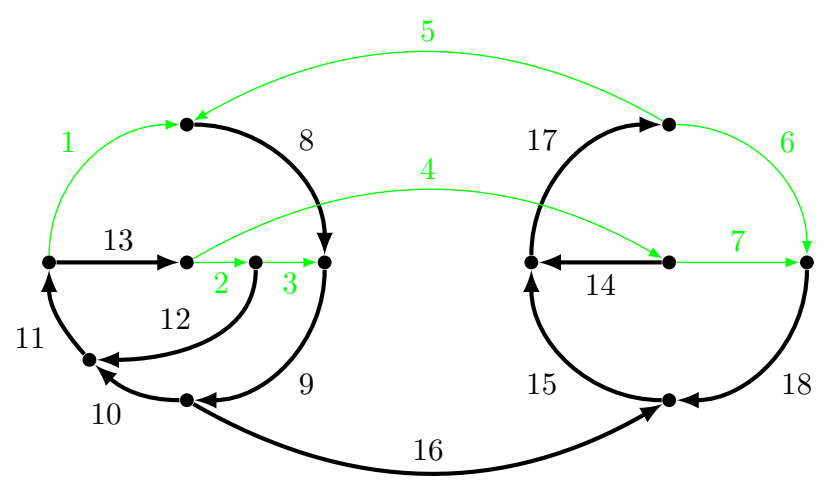

Figure 13. The Graph $G_{4}$

$$
\begin{aligned}
q_{G_{4}}\left(x_{1}, x_{2}, x_{3}, x_{4}, x_{5}, x_{6}, x_{7}\right)= & x_{1}^{2}-x_{1} x_{4}-x_{1} x_{5}-x_{1} x_{7}+x_{2}^{2}+ \\
& x_{2} x_{3}-x_{2} x_{4}-x_{2} x_{5}+x_{3}^{2}-x_{3} x_{4}- \\
& x_{3} x_{5}+x_{4}^{2}+x_{4} x_{5}+x_{4} x_{7}+x_{5}^{2}- \\
& x_{5} x_{6}+x_{5} x_{7}+x_{6}^{2}-x_{6} x_{7}+x_{7}^{2}
\end{aligned}
$$

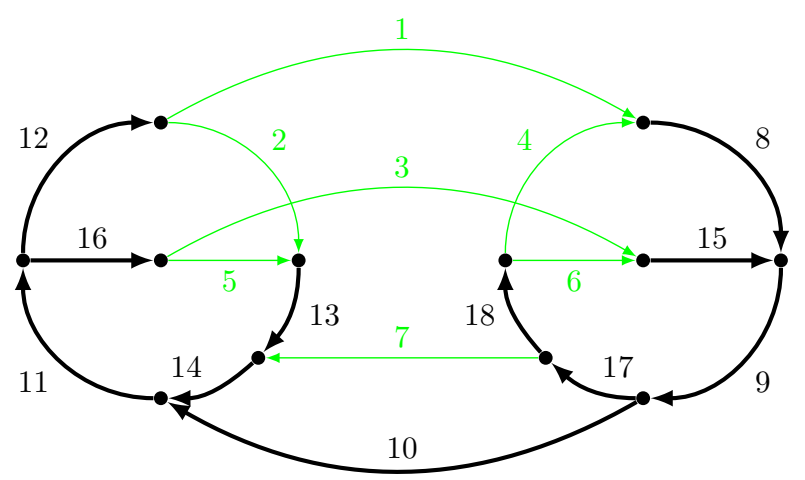

Figure 14. The Graph $G_{5}$

$$
\begin{aligned}
q_{G_{5}}\left(x_{1}, x_{2}, x_{3}, x_{4}, x_{5}, x_{6}, x_{7}\right)= & x_{1}^{2}-x_{1} x_{2}-x_{1} x_{4}+x_{1} x_{7}+x_{2}^{2}- \\
& x_{2} x_{5}+x_{3}^{2}-x_{3} x_{5}-x_{3} x_{6}+x_{3} x_{7}+ \\
& x_{4}^{2}-x_{4} x_{6}+x_{5}^{2}+x_{5} x_{6}-x_{5} x_{7}+ \\
& x_{6}^{2}-x_{6} x_{7}+x_{7}^{2}
\end{aligned}
$$




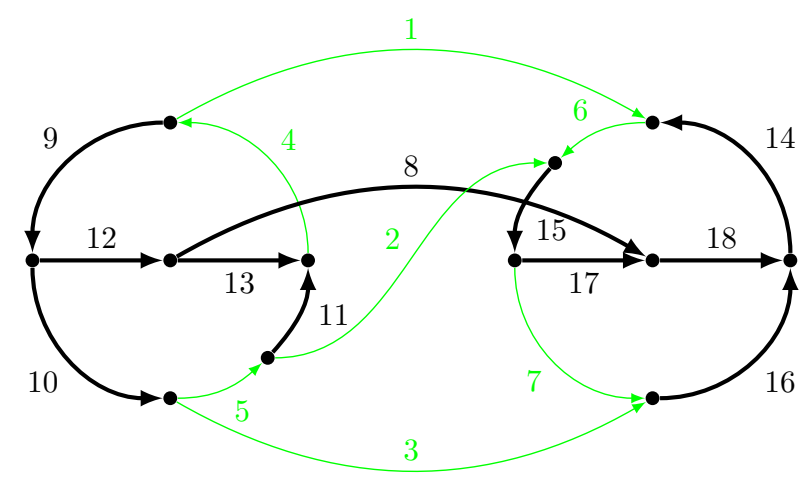

Figure 15 . The Graph $G_{6}$
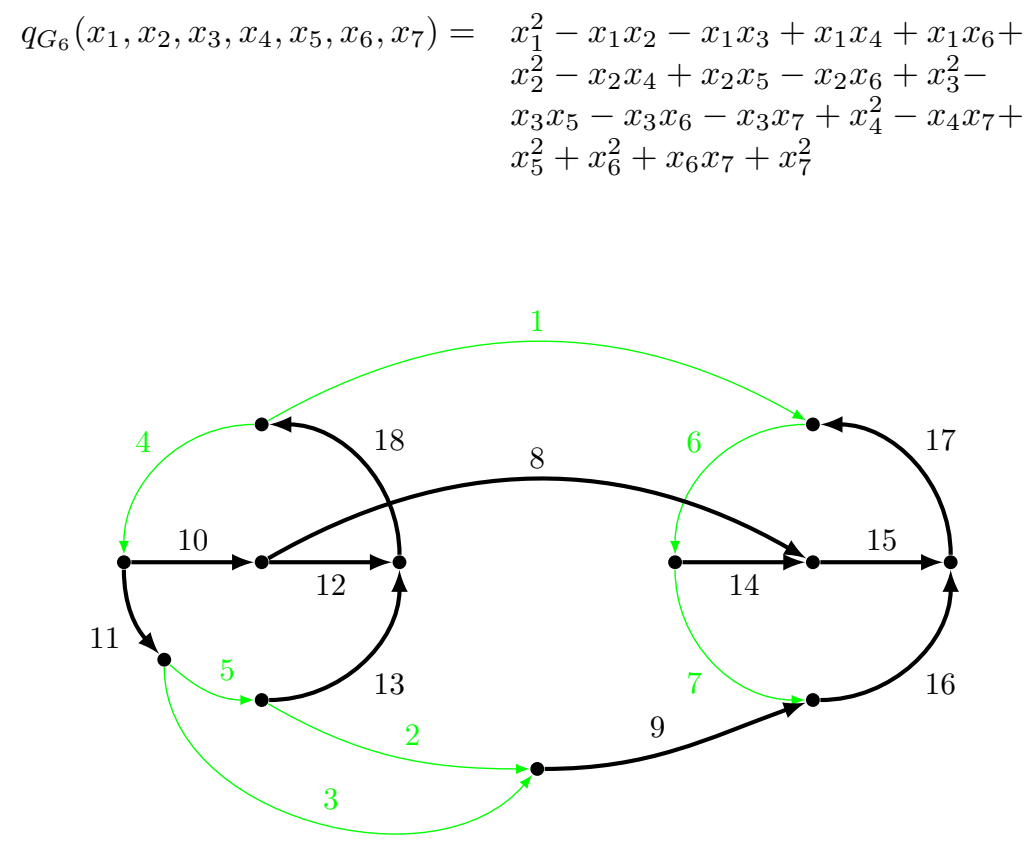

Figure 16 . The Graph $G_{7}$

$$
\begin{aligned}
q_{G_{7}}\left(x_{1}, x_{2}, x_{3}, x_{4}, x_{5}, x_{6}, x_{7}\right)= & x_{1}^{2}-x_{1} x_{2}-x_{1} x_{4}-x_{1} x_{5}+x_{1} x_{6}+ \\
& x_{2}^{2}-x_{2} x_{3}+x_{2} x_{5}-x_{2} x_{6}-x_{2} x_{7}+ \\
& x_{3}^{2}-x_{3} x_{5}+x_{4}^{2}+x_{4} x_{5}-x_{4} x_{6}+ \\
& x_{5}^{2}-x_{5} x_{6}+x_{6}^{2}+x_{6} x_{7}+x_{7}^{2}
\end{aligned}
$$




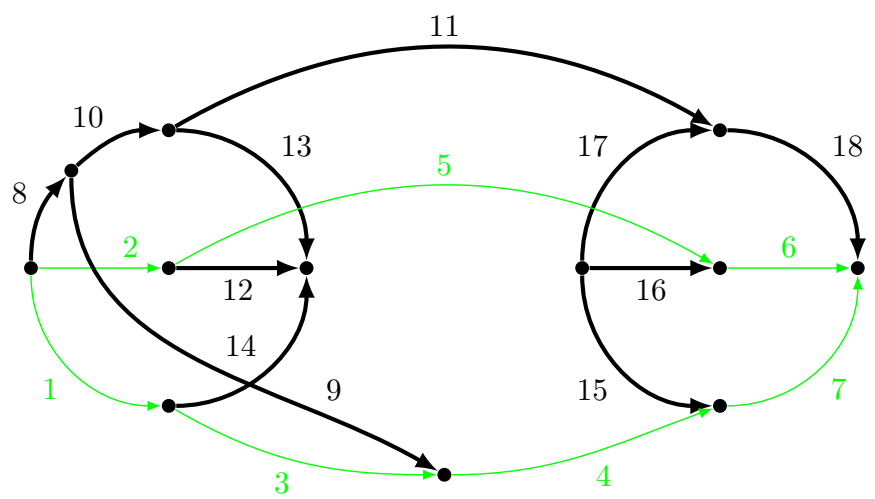

Figure 17. The Graph $G_{8}$
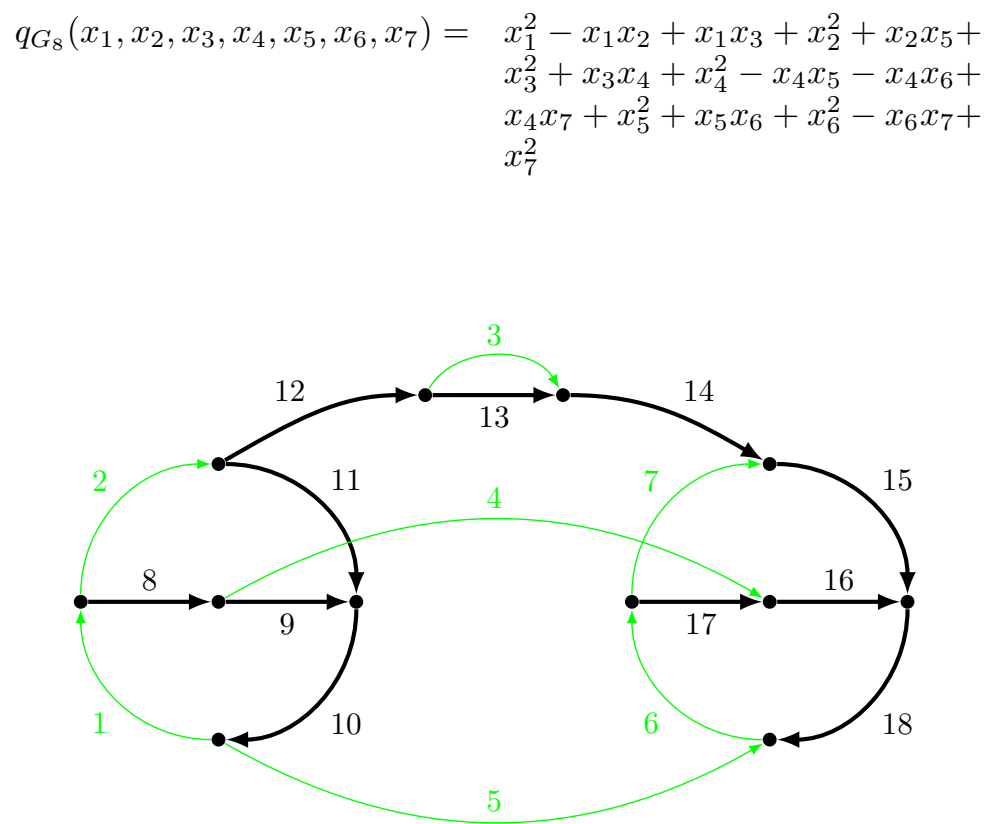

Figure 18. The Graph $G_{9}$

$$
\begin{aligned}
q_{G_{9}}\left(x_{1}, x_{2}, x_{3}, x_{4}, x_{5}, x_{6}, x_{7}\right)= & x_{1}^{2}+x_{1} x_{2}+x_{1} x_{4}-x_{1} x_{5}+x_{2}^{2}+ \\
& x_{2} x_{3}-x_{2} x_{5}+x_{3}^{2}-x_{3} x_{4}+x_{3} x_{6}+ \\
& x_{4}^{2}-x_{4} x_{5}-x_{4} x_{6}+x_{5}^{2}+x_{5} x_{6}+ \\
& x_{5} x_{7}+x_{6}^{2}+x_{6} x_{7}+x_{7}^{2}
\end{aligned}
$$




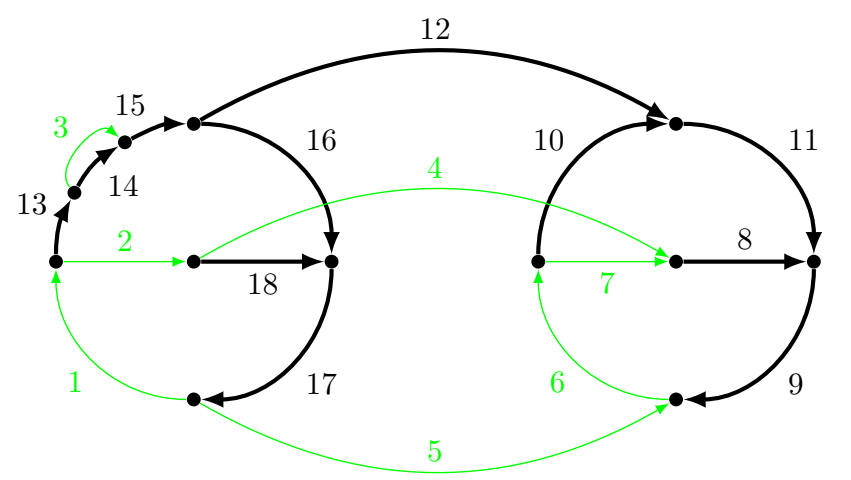

Figure 19. The Graph $G_{10}$

$$
\begin{aligned}
q_{G_{10}}\left(x_{1}, x_{2}, x_{3}, x_{4}, x_{5}, x_{6}, x_{7}\right)= & x_{1}^{2}+x_{1} x_{2}+x_{1} x_{3}+x_{1} x_{4}-x_{1} x_{5}+ \\
& x_{2}^{2}+x_{2} x_{4}+x_{3}^{2}+x_{4}^{2}-x_{4} x_{5}- \\
& x_{4} x_{7}+x_{5}^{2}+x_{5} x_{6}+x_{5} x_{7}+x_{6}^{2}+ \\
& x_{6} x_{7}+x_{7}^{2}
\end{aligned}
$$

\section{Genus 8}

As we explained in Section 2.3, it is sufficient to find a $\mathbb{Z}$-emm for each of the finitely many graphs obtained from $F_{11}-F_{14}$ and $G_{1}-G_{10}$ by applying procedure (a) or (b). This gives $14 \cdot\left(\left(\begin{array}{c}18 \\ 2\end{array}\right)+18\right)=2394$ graphs.

We have written a Mathematica program for computing the $8 \times 13$ matrices for these graphs, and a Fortran program which uses integer arithmetics for finding the $\mathbb{Z}$-emms. We confirmed that they exist for all of these graphs. The lists of the matrices and the $\mathbb{Z}$-emms are available at http://www. math .uga. edu/ valery/vigre2010.

\section{References}

[AB11] Valery Alexeev and Adrian Brunyate, Extending Torelli map to toroidal compactifications of Siegel space, arXiv:1102.3425 (2011), 1-16.

[Arc80] Dan Steven Archdeacon, A Kuratowski theorem for the projective plane, ProQuest LLC, Ann Arbor, MI, 1980, Thesis (Ph.D.)-The Ohio State University.

[Arc81] Dan Archdeacon, A Kuratowski theorem for the projective plane, J. Graph Theory $\mathbf{5}$ (1981), no. 3, 243-246.

[GH75] Henry H. Glover and John P. Huneke, Cubic irreducible graphs for the projective plane, Discrete Math. 13 (1975), no. 4, 341-355.

[GHW79] Henry H. Glover, John P. Huneke, and Chin San Wang, 103 graphs that are irreducible for the projective plane, J. Combin. Theory Ser. B 27 (1979), no. 3, 332-370.

[Igu67] Jun-ichi Igusa, A desingularization problem in the theory of Siegel modular functions, Math. Ann. 168 (1967), 228-260.

[Mil73] Martin Milgram, Irreducible graphs. II, J. Combinatorial Theory Ser. B 14 (1973), $7-45$.

[Nam73] Yukihiko Namikawa, On the canonical holomorphic map from the moduli space of stable curves to the Igusa monoidal transform, Nagoya Math. J. 52 (1973), 197-259. 
Department of Mathematics, University of Georgia 